\title{
Plurilinguisme du roman chinois francophone et approche de la diversité et de la pluralité en FLE
}

\author{
Béatrice BOUVIER-LAFFITTE \\ UCO-Licia, Angers ; CoDiRe, EA 4643, Université de Nantes, France
}

Il n'est pas de langue qui ne soit une langue-mixte, il n'est pas de subjectivité qui ne soit composite. Jean-Marie Prieur

\section{Introduction}

Les écrivains qui, comme Édouard Glissant «ne sont pas installés dans la masse tranquille de leur langue », pratiquent des formes d'écriture plurilingue et pluriculturelle. En effet, ils mobilisent les langues de leur répertoire pour agir dans le monde social, s'y intégrer et produire un langage singulier qui tisse l'homogène avec l'hétérogène selon les termes du projet plurilingue imaginé par le Conseil de l'Europe (2001).

Cette forme de plurilinguisme n'est pas synonyme de multilinguisme; alors que ce dernier est plutôt associé à la notion de juxtaposition ou de cohabitation des langues, le plurilinguisme européen défend l'idée « d'une complémentarité des acquis de l'individu dans les différentes langues connues, le tout constituant une compétence plurilingue et pluriculturelle unique et complexe »(Candelier 37). De même, la compétence plurilingue n'est pas l'équivalent de la compétence polyglotte; il ne s'agit pas de compétences juxtaposées mais plutôt d'une compétence plurielle, partielle, composite. Cette compétence plurilingue (CP) est comprise comme globale, dynamique, évolutive, singulière et porteuse de marques symboliques et identitaires. Détenteur d'un répertoire linguistique singulier qu'il s'agit de développer et de rendre opérationnel, chaque sujet peut alors être considéré comme fondamentalement pluriel.

Nous observerons à travers un corpus composé d'extraits autobiographiques et de fragments de romans de quelle manière et dans quelle mesure ces écrivains chinois d'expression française exercent leur compétence plurilingue, définie comme une pratique qui «englobe l'ensemble du répertoire langagier à disposition et le dote d'une compétence communicative à laquelle contribuent toute connaissance et toute expérience des langues et dans laquelle les langues sont en corrélation et interagissent »(Beacco et Byram 11). Il s'agira d'observer comment s'opère la construction langagière de soi et du monde en français à partir d'un répertoire constitué d'au moins deux langues, en l'occurrence le chinois et le 
français. Actuellement neuf écrivains chinois composent et publient en français ${ }^{1}$. S'agissant de cet article notre corpus inclura des œuvres de Ying Chen, François Cheng, Dai Sijie, et Shan Sa.

\section{Inscription dans une problématique interculturelle}

Le choix de travailler sur des textes ou œuvres exclusivement issus des littératures chinoises francophones n'est pas sans effets pour la réflexion didactique développée ici. Cette littérature est actuellement peu connue, peu travaillée, y compris par les spécialistes de la littérature francophone. Ce domaine littéraire, circonscrit à une aire linguistique et géographique lointaine, n'appartient pas à la francophonie contrainte, celle des anciens pays colonisés; il est peu ou pas représenté dans les manuels de FLE ni dans les ouvrages spécialisés en didactique de la littérature et peut apparaitre comme n'ayant pas de rapport direct avec la culture française, contrairement aux littératures francophones de l'aire du Maghreb ou de l'Afrique qui ont de nombreux liens historiques, sociaux, passés et contemporains avec la langue et la culture françaises. On notera que cette absence de liens sociolinguistiques vaut aussi dans l'autre sens et que ces auteurs sont, pour ces mêmes raisons, coupés d'un éventuel lectorat compatriote, et ils sont de ce fait, ainsi que pour des raisons idéologiques, privés de toute reconnaissance dans leur pays d'origine ${ }^{2}$.

Par leurs écrits, leur parcours individuel, ces auteurs apportent de nouvelles formes de métissage, jouent et déjouent avec les appartenances, les variations linguistiques et identitaires pour redessiner, non seulement les contours de la francophonie littéraire, mais aussi ceux du métissage, de l'hybridation, de l'acculturation, de l'entre-langues.

Leur écriture romanesque est occupée de l'intérieur par l'histoire et la géographie ; mais elle l'est aussi par leur répertoire linguistique. Ces textes nous proposent de nouveaux espaces dont il s'agira d'identifier l'altérité qui se présente peut-être sous des formes autres que celles qui sont déjà signifiantes ou observées en didactique du FLE ou qui, et c'est notre hypothèse, sont susceptibles de favoriser une approche de la pluralité, celle des interprétations, celle des langues et de la diversité linguistique et culturelle pour les apprentissages en FLE.

Un cheminement à travers la biographie et les textes des protagonistes de cette étude nous amènera à observer une certaine forme de problématisation de la langue, en particulier dans le rapport à la langue française ainsi que la mise en œuvre d'un plurilinguisme textuel dont nous chercherons à élucider les mécanismes. 


\section{Biographies métissées et apprentissage des langues}

Parmi toutes les conséquences liées à la condition d'exilé, on remarquera plus particulièrement chez les protagonistes de cette étude le changement de langue (passage du chinois au français) ainsi que le début de la création littéraire qui est venue s'ajouter, seulement après l'installation en pays francophone, aux activités pratiquées antérieurement en langue chinoise. Le changement de langue n'est ni neutre, ni anodin ; le passage du chinois (mandarin et/ou dialecte régional) au français qui s'opère à l'âge adulte, coïncide avec des ruptures, engendre des bouleversements identitaires qui conduisent à questionner, penser et repenser les liens entre langues et identités. Ying Chen, François Cheng et Shan Sa associent clairement l'entrée dans la langue nouvelle à une renaissance : Ying Chen «se glisse dans une autre langue et espère y renaitre »; et elle affirme qu'il lui aurait été impossible d'écrire sans avoir quitté la Chine (Encres vagabondes). Shan Sa poursuit cette idée de renouvèlement: «Écrire en français c'était tourner définitivement la page (celle des évènements de la Place Tian Anmen) » (Double Je) que l'on retrouve aussi chez François Cheng, homme sans parole pendant vingt ans qui explique : «Puis, petit à petit, immergé dans cette terre d'accueil et initié à cette nouvelle langue, j'ai éprouvé l'ivresse de renommer les choses à neuf comme au matin du monde » (Entretien Argand, 2001). Ce changement de langue, qui intervient tardivement, a nécessité un long, douloureux apprentissage du français dont on trouve de multiples témoignages dans les textes biographiques ou récits de ces écrivains. François Cheng, aujourd'hui académicien français, développe longuement cette question de l'apprentissage de la langue :

Vers la fin des années 50, j’ai été pris de découragement ; j'étais en perdition car je ne pouvais ni m'exprimer en français puisque je ne possédais pas suffisamment cette langue, ni en chinois faute d'avoir la possibilité de me replonger dans la réalité de mon pays. Ma culture d'origine et l'art chinois n'avaient pas la notoriété qu'ils ont aujourd'hui. (Dialogue, 26)

Les auteurs du corpus présentent des parcours d'apprentissage variés et originaux ; ils ont déployé, pour acquérir le français, une large gamme de stratégies d'apprentissage dont ils témoignent volontiers. Ces « auteurs FLE », comme les appelle Anne-Rosine Delbart (145), sont les grands prédécesseurs des apprenants sur le chemin de la maitrise du français, ils « révèlent la variété des conditions et des situations d'apprentissage, les handicaps mais aussi les avantages du regard autre porté sur la langue cible. Ils montrent combien la force de la motivation permet de combattre les difficultés d'un apprentissage tardif ». Shan Sa compare ses premières phrases de composition française à du gruyère, avec des trous qu'elle comblait de mots traduits du chinois «[...] mais ce n'était pas bon bien évidemment, je n'avais pas la 
musique de la langue française, et il y a eu une très longue attente, et aujourd'hui, il y a une urgence à s'exprimer $\gg($ Double $\mathrm{Je})$.

Les expériences d'apprentissage du français relatées par ces écrivains sont de précieux outils de réflexion; ils permettent aux apprenants de comparer leurs difficultés, leur motivation dans le choix et dans l'apprentissage du français avec le parcours de ces étrangersécrivains ; de progresser en cheminant entre identification et autonomie : «Ils sont la preuve qu'une langue apprise peut être vivante, attractive, ludique, libre de s'épanouir dans le carcan de la norme vécue comme une protection et capable de briser le moule rigide des idées reçues » (Delbart145).

La réflexion autour de l'apprentissage d'une langue étrangère, devenue langue seconde puis langue d'écriture, apporte un éclairage à la question des interrelations entre langues, de l'interaction entre langues et création et nous donne les moyens de reposer la question de la langue dans le rapport pluriel et dialectique qu'elle entretient avec l'identité :

[...] je rêvais, je rêve encore de franchir la barrière des langues, convaincue que toutes les cultures peuvent me nourrir, que je suis ma propre origine qui se forme et se reforme au fur et à mesure que je voyage, que je suis moi avant d'être shanghaienne, chinoise, québécoise, canadienne ou autre. (Ying Chen, Marches, 42)

D'ailleurs, Martine Abdallah-Pretceille a souvent souligné l'intérêt de travailler sur les biographies métissées dans les parcours ou dans les productions littéraires « afin de mettre en relief cette sociologie de la bigarrure qui est celle de la modernité » (Abdallah-Pretceille et Porcher 159 ; Abdallah-Pretceille 151).

\section{Du plurilinguisme linguistique au plurilinguisme textuel}

Les auteurs du corpus (François Cheng, Ying Chen, Dai Sijie et Shan Sa) font preuve d'inventivité langagière et écrivent un français dans lequel d'autres langues (chinois mandarin, dialectes chinois) occupent une place ; ils expriment leur imaginaire poétique et sonore grâce à l'intertextualité mais aussi aux jeux des résonances, assonances, allitérations, métaphores, emprunts lexicaux, ponts-verbaux... Pour François Cheng, «La langue maternelle faite d'images et de signes figuratifs révèle la vibration du français ; vocalise le mot, sonorise les mots ; approche stéréophonique ou stéréoscopique » (Dialogue, 79).

Le plurilinguisme littéraire ou textuel se définit comme la cohabitation de plusieurs langues dans un texte romanesque qui se présente, au premier abord, comme unilingue. Pour traiter ce phénomène de plurilinguisme textuel, R. Grutman emploie le terme de diglossie. Une diglossie qui se manifestant à l'intérieur d'un texte en français « devient une sorte de 
palimpseste portant les traces d'une écriture première, dans la langue de l'auteur : calques créant un effet de polyphonie, intercalation de genres oraux, travail sur le signifiant sont quelques-unes des formes que prend l'inscription littéraire de la (ou des) langue(s) dominée(s) » (61). Pour D. Maingueneau, l'écrivain n'est pas confronté à la langue mais à l'interaction de langues et d'usages et il identifie ce processus comme une interlangue « définie comme le résultat des variations au sein d'une même langue mais aussi entre cette langue et les autres » (104). Il revient plus tard sur les deux formes de plurilinguisme initialement développées : le plurilinguisme externe, qui gère la relation des œuvres aux «autres » langues, ou le plurilinguisme interne, c'est-à-dire la diversité au sein d'une même langue pour conclure « qu'en définitive, c'est l'œuvre qui trace la frontière entre l'intérieur et l'extérieur de «sa » langue »(140-141).

Shan Sa a mis des années avant d'entendre «la musique du français »; mais elle choisit une attitude assez revendicative et traite son bilinguisme comme un outil de travail, une marque stylistique et identitaire, en déclarant : «Et j'espère que cette langue française est écrite de telle manière qu'à travers elle, on aperçoit ce qu'est la langue chinoise. C'est peutêtre là ce qui fait le style de tous mes livres »(Double Je).

Chez ces écrivains, les langues, le chinois et le français ne sont pas en conflit, il s'agirait plutôt d'un bilinguisme conciliant, opérationnel, ce qui ne signifie pas qu'il s'est construit sans souffrance, ni doutes ni espérances déçues, mais ce qui transparait dans l'écriture romanesque tend vers un tissage dans lequel les langues se croisent, s'entrelacent, se répondent, se font écho. On pourrait même parler d'entre-aide, le chinois venant au secours d'une création langagière qui se trouve bien souvent engloutie dans les méandres du délicat travail d'appropriation de la langue d'écriture, le français : «Dans mes écrits, que ce soit dans ma poésie ou bien dans mes romans, toute la sensibilité, toutes les images que véhicule le chinois continuent bien sur à m'alimenter », nous dit Cheng (Entretien Argand, 2001). Les deux langues sont complémentaires, elles composent les différentes facettes d'un langage en perpétuel devenir et Ying Chen, même lorsqu'il s'agit de s'auto-traduire, refuse obstinément de choisir entre l'une ou l'autre et considère l'œuvre comme une version originale dans chacune des langues. Elle craint même de perdre la langue chinoise «cet héritage facile à recevoir, difficile à garder » (Marches, 70) et ressent un tel vertige à l'idée de perdre l'une ou l'autre langue qu'elle envisage d'en ajouter une troisième à son répertoire : «Tout cela m'incite à m'accrocher le plus longtemps et le mieux possible à la langue chinoise autant qu'à la langue française, et possiblement à une autre langue encore » (71). 
Dans ces exemples, l'interaction entre les langues s'organise autour d'une alternance considérée comme une ressource; elle repose sur la représentation dynamique d'un plurilinguisme vécu non pas comme une situation stable et figée mais bien comme un processus en construction :

La langue française est cette pierre qui quelquefois m'échappe, d'autres fois me réconforte, mais jamais ne m'appartiendra de façon absolue. Les mots se moquent de moi et les phrases se décomposent dans ma tête. Il me faut alors revenir dans les dictionnaires et tout recommencer. [...] Non seulement l'écriture dans une langue seconde est un travail digne de Sisyphe, mais la création en général l'est également. (27)

On voit combien de telles expériences des langues contribuent au développement d'une conscience linguistique indispensable à l'élaboration d'une compétence plurilingue et pluriculturelle différenciée.

La langue d'écriture de ces auteurs s'exprime dans un français standard et, à l'exception de quelques mots en chinois du registre onomastique ou gastronomique, elle peut sembler, à première vue, peu marquée du sceau de l'origine chinoise. Pourtant, dans l'épreuve de la création, la langue construit sa singularité faite d'associations d'idées, de rythmes et de sonorités dont l'auteur bilingue expérimente le pouvoir innovant et poétique.

\section{Titres et premières de couvertures}

Les premières de couverture des romans du corpus fournissent des supports évocateurs qui sont non seulement propices à la création d'un horizon d'attente, mais sont aussi porteurs d'altérité linguistique et culturelle. L’iconographie soignée, photo, peinture ou calligraphie, peut être soumise aux hypothèses et à l'imagination des apprenants avant d'entrer proprement dit dans la lecture ${ }^{3}$. On peut observer, par exemple, la présence d'un sceau à la cire rouge sur la première de couverture de L'éternité n'est pas de trop. En dehors de son esthétisme, peut-être de son exotisme pour le lecteur francophone, le sceau apporte des informations concrètes; il est d'ordinaire une signature, celle du peintre, du propriétaire, d'une dynastie etc., mais en l'occurrence celui-ci est composé des deux caractères shan et shui (montagne-eau) autrement dit peinture de paysage qui est le genre auquel appartient cette peinture traditionnelle. Un genre imprégné de taoïsme qui valorise les éléments naturels et rappelle à l'homme sa modeste condition à l'échelle de l'univers. Cette illustration n'a pas été choisie à la légère par Cheng, spécialiste d'art pictural chinois ${ }^{4}$ et elle constitue une bonne introduction à ce roman qui ne manque pas de convoquer les éléments naturels pour traduire 
la portée cosmique des sentiments humains ${ }^{5}$ : «Astre à astre, cœur à cœur ! Ces étoiles sontelles différentes des humains sur la terre ?» (166).

\section{Interférences de langues et poétiques narratives}

La phrase en chinois est constituée d'une suite d'éléments juxtaposés dont seuls l'ordre et le positionnement dans la phrase permettent au lecteur d'élaborer des relations significatives entre eux. Les nuances, les articulations, les précisions, les relations de cause à effet se font par oppositions/contrastes. Les mots, côte à côte, se mettent en valeur, se contredisent, s'opposent, se répondent grâce au jeu des redondances, des répétitions, des variations sur un même mot. C'est un langage figuratif et imagé qui n'explique pas mais évoque et propose.

Observons dans le détail la mise en œuvre du bilinguisme textuel de Cheng dans l'extrait suivant issu de L'Éternité n'est pas de trop :

La corole à cinq pétales, quand elle éclot, est un gant retourné de l'intérieur vers l'extérieur, elle livre son fond secret, se laisse effleurer par la brise tiède qui sans cesse passe, ou butiner sans fin par d'avides papillons et abeilles qui accourent. Entre deux mains aux doigts noués, le moindre frémissement bruit de battements d'ailes ; la moindre pression provoque une onde qui s'élargit de cercle en cercle. La main, ce digne organe de la caresse, ce qu'elle caresse ici n'est pas seulement une autre main, mais la caresse même de l'autre. Caressant réciproquement la caresse, les deux partenaires basculent dans un état d'ivresse qui a peut-être été rêvé dans l'enfance, ou alors dans une avant-vie. (85)

En ce qui concerne la répétition, la redondance et les variations sur un même mot, voici ce que nous offre ce court passage : le terme «main» est répété trois fois, puis à nouveau désigné par deux métaphores (La corole à cinq pétales, un gant retourné de l'intérieur vers l'extérieur) et enfin par une paraphrase (La main, ce digne organe de la caresse). On remarquera la grande sensualité qui se dégage de ces métaphores dans un récit par ailleurs très pudique. Les éléments de la nature (la brise, l'onde, les papillons et les abeilles) avec leur bruissement d'eau, de vent ou leurs battements d'ailes, inscrivent dans un paysage sonore le sens le plus sollicité de ce passage ; le toucher, lui-même marqué par une isotopie explicite « frémissement », «pression », « caressant » et « caresse ».

Le terme de «caresse» est décliné pas moins de quatre fois sous des formes différentes allant du substantif au verbe en passant par le participe présent (la caresse, caresse, caressant). Enfin, on remarquera aussi le jeu d'assonance sur le son «-esse » (cesse, caresse, ivresse) qui égrène sa musique légère et caressante tout au long du passage. 
Le foisonnement d'images issues de la nature donne une dimension poétique à l'expression de Cheng ainsi qu'une portée universelle et intemporelle comme l'annonce d'emblée le titre du roman : L'éternité n'est pas de trop. D'ailleurs pour Cheng « La nature n'est pas un décor mais la part vitale de notre esprit. La nature nous initie à la beauté et à la vie tout en incarnant ce que notre esprit porte en lui »(Cinq méditations, 32).

\section{L'homophonie d'une langue à l'autre}

Dans un tout autre registre Dai Sijie pratique une mise à distance ironique et convoque la fonction ludique et poétique du plurilinguisme textuel sur le terrain de l'onomastique. Les jeux de noms sont tout à la fois lieu d'échange avec le lecteur, à qui il aime donner des explications, et de correspondances phoniques entre les deux langues. C'est à travers l'usage des noms, prénoms et appellatifs que les fragments étudiés nous révèlent ce substrat de langue chinoise propre au plurilinguisme textuel.

L'homophonie est une des caractéristiques de la langue chinoise (un grand nombre de mots de sens différents ont une prononciation proche ou identique) et constitue un des terreaux les plus fertiles de cette langue pour les jeux humoristiques fondés sur les contrepèteries, les confusions, les contresens, les malentendus et autres quiproquos. Dans le titre de son roman Le Complexe de Di, Dai Sijie fait jouer l'homophonie entre deux langues, le français «Le Complexe d'Edipe» et le chinois «Le Complexe de Di»; ce titre fonctionne comme un calque syntaxique, morphologique et homophonique, ou bien comme un palimpseste bilingue : Edipe et $D i$ sont deux noms propres dont on entend bien le lien phonique même s'il est limité à une seule unité syllabique en raison du monosyllabisme des noms propres chinois. Ce jeu d'homophonie annonce le thème du roman (l'introduction de la psychanalyse en Chine) d'une façon voilée car c'est un jeu de mots qui s'entend plus qu'il ne se lit.

Ce palimpseste phonique cache une autre référence plus culturelle, celle à un personnage connu ; le juge Ti (Di ou Ti, selon la transcription phonétique choisie), héros de la saga Les Enquêtes du juge $T^{6}{ }^{6}$. Ce juge Ti, inspiré d'un magistrat chinois ayant réellement existé, a permis dès les années cinquante aux lecteurs occidentaux de se plonger dans une Chine ancienne décrite avec rigueur et réalisme par la plume experte de Robert Van Gulick.

Mais Dai Sijie, ne s'arrête pas là et cite dans ses romans bon nombre de noms d'auteurs ou de personnages de la littérature française, tantôt traduits tantôt expliqués: «Cristo, c'est un autre nom de Jésus, qui veut dire le messie, ou le sauveur » (Balzac, 130); 
«Le Père Goriot dont le titre chinois était le Vieux Go » (113). Il les agrémente parfois d'un titre, à la manière chinoise : « Maitre Dumas » (130).

L’usage du nom de famille en chinois est privilégié à celui du prénom ; son emploi, avec ou sans titre, est toujours approprié quel que soit le contexte, contrairement au prénom qui, lui, est d'un maniement plus délicat, plus restreint. La tradition chinoise considère comme important de donner un prénom poétique, positif et, si possible, unique à chaque nouveau-né ; tous les mots de la langue sont disponibles pour la création de ce prénom qui a donc toujours une signification ce qui lui confère un caractère très personnel, intime. Le potentiel d'évocation du prénom, doublement servi et par sa graphie et par sa prononciation, se révèle dans ce passage de Les Lettres chinoises : «Chaque fois que je prononce Sassa $^{7}$, je pense à la chaleur du sable, à la sonorité des soupirs, à la gaieté des pieds nus, à la folie du vent, à l'éternité du soleil et de la mer » (Ying Chen 57).

Dai Sijie se joue de la tradition chinoise concernant les prénoms; il n'hésite pas à les tourner en ridicule soit par le choix de termes impropres «Volcan de la vieille lune » (volcan n'est pas suffisamment féminin et vieille pas du tout positif), soit par une accumulation de compléments du nom tout à fait contraire à la règle chinoise, «La Princesse de la montagne du Phénix du Ciel ». On peut se demander si la moquerie cible les prénoms chinois euxmêmes ou les traductions qui en sont parfois faites en français au moyen de périphrases plus ou moins heureuses. Quoiqu'il en soit, ces jeux de noms sont des occasions de s'interroger sur les différentes formes de dénomination, celles en pratique dans sa propre culture, celles en vigueur ailleurs. Mais c'est aussi une ouverture pour travailler la langue à partir du pouvoir évocateur de certains mots, de leur potentiel poétique.

Dans Balzac et la Petite Tailleuse chinoise, les personnages sont parfois présentés par un surnom, comme fréquemment en chinois, «La Petite Tailleuse », «Le Binoclard », « Le Vieux Tailleur ». Enfin les nom et prénom du narrateur nous sont livrés sous forme de rébus «trois dessins représentant l'un un cheval, l'autre une longue épée pointue et le dernier une petite clochette qui tinte » (115). Cette forme de présentation est suffisamment précise et habituelle pour qu'un lecteur chinois identifie rapidement les sinogrammes qui composent le nom et soit ainsi à même d'en reconstituer la graphie et la prononciation. On notera cependant que cette formulation, même si elle pique son imagination, reste hermétique pour le lecteur francophone qui est, malgré tout, le premier destinataire du roman. Mais n'est-ce pas là la volonté de l'auteur ? 
Grâce à cette mise en scène onomastique, Dai Sijie transmet, compare, explique des usages chinois; et au fil du récit, des traces sonores, graphiques (le rébus) de la langue chinoise souterraine s'inscrivent dans l'écriture en français.

L'écriture de Ying Chen se caractérise par une recherche de concision et d'économie de mots qui engendre un style limpide et dépouillé ; une énonciation simple et concise, des phrases courtes qui nous rappellent qu'en chinois la concision, érigée en vertu, fait la beauté de la langue. L'enracinement dans la langue natale se manifeste aussi par une intertextualité riche et variée, marquée par l'insertion dans le corps du récit de dictons, de proverbes, de chansons, de légendes, de poèmes traduits du chinois, comme celui que choisit Yuan pour écrire à son amoureuse Sassa combien elle lui manque :

Comme le dit un vieux poème :

Nos larmes coulent dans la solitude

Le ciel est loin et la terre immense

Inutile de chercher ici et là

Nous n'aurons plus d'ancêtres ni d'enfants. (Lettres chinoises, 58)

Ce court poème exprime mieux qu'un long discours le sentiment de solitude éprouvé par le personnage ; cette respiration poétique insérée au milieu de la narration est aussi un subtil rappel de l'art poétique de la période Tang qui, en une ou plusieurs strophes de quatre vers, traite de thèmes tels que la solitude, le temps qui passe, la séparation d'avec les êtres chers ou les consolations apportées par le vin ou l'amitié.

\section{Conclusion}

Le texte de littérature chinoise francophone offre la possibilité de travailler avec la pluralité des interprétations et des savoirs comme avec la pluralité des langues. En effet, nous avons observé comment un texte qui se présente en français standard, établit avec une autre langue, le chinois, des ponts, des liens, des articulations. Cette interlangue, en créant un réseau associatif qui opère au travers d'assonances, d'homophonies, de métaphores, donne à voir un peu, beaucoup ou passionnément de cette langue, de cette culture initiale qui s'invite là où on ne l'attend pas. Par ailleurs, la problématisation et la réflexivité exprimées dans les œuvres en ce qui concerne l'appropriation et l'apprentissage du français, sont autant de manières «d'être aux langues » qui peuvent contribuer au développement d'une éducation à la diversité et à la pluralité. Nous n'avons exploré, dans cette étude, que quelques-unes des manifestations d'un plurilinguisme textuel qui, dans ces textes comme dans d'autres, peut être envisagé comme un territoire plurilingue, pluriculturel au service du développement de la capacité à «mettre en œuvre, en situation et dans l'action, un répertoire constitué de 
ressources plurielles et diversifiées qui permet de se reconnaitre et de s'affirmer en tant qu'acteur social plurilingue » (Moore et Castellotti 18). Et si, comme l'affirme Cheng, «Tout est toujours nouveau par ce qui nait 'entre' »(Dialogue : 72), alors envisageons les multiples singularités de ces écritures comme des ressources et comme des moyens dont l'enjeu serait la démarche d'élucidation et non pas l'aboutissement.

\section{Corpus}

François Cheng. L'éternité n'est pas de trop. Paris : Albin Michel, 2002.

Dai Sijie. Balzac et la petite tailleuse chinoise. Paris : Gallimard, 2000.

---. Le complexe de Di. Paris : Gallimard, 2003.

Shan Sa. La joueuse de go. Paris : Grasset, 2001

---. Les quatre vies du saule. Paris : Grasset, 1999

Ying Chen. Les lettres chinoises. Paris : Actes Sud/Léméac, 1993

---. Quatre mille marches. Un rêve chinois. Paris : Seuil, 2004

\section{Bibliographie}

Abdallah-Pretceille, M., 2010. «La littérature comme espace d'apprentissage de l'altérité et du divers ». Synergies Brésil nº spécial. 145-155.

--- et L. Porcher. Éducation et communication interculturelle. Paris : PUF, 1996.

Beacco, J-C. et M. Byram. 2003. Guide pour l'élaboration des politiques linguistiques éducatives en Europe. De la diversité linguistique à l'éducation plurilingue. Strasbourg : Conseil de l'Europe. Disponible en ligne. http://www.coe.int.

Candelier, M. «Cohésion sociale, compétence plurilingue et pluriculturelle : quelles didactiques ». Les langues modernes, Revue de l'APLV 4 (2005). 35-45.

Cheng, François. Le Dialogue. Une passion pour la langue française. Desclée de Brouwer, P. littéraires et artistiques de Shanghai, 2002.

Delbart A.-R., 2005. «Un atout pour la construction d'une conscience linguistique de la langue cible chez les apprenants de français langue étrangère : l'exemple des écrivains venus d'ailleurs ». GLOTTOPOL, «Construction de compétences plurielles... » 6 (2005). 134-145. http://www.univ-rouen.fr/dyalang/glottopol [consulté le 19/07/2012]

Grutman, R. «Diglossie littéraire». Vocabulaire des études francophones. Les concepts de base. Limoges : PU de Limoges, 2005.

Maingueneau, D. Éléments de linguistique pour le texte littéraire. Paris : Dunod, 1993. 
Moore, D. et V. Castellotti. La compétence plurilingue : regards francophones. Fribourg: Lang, collection Transversales, 2008.

Shan Sa, Double Je. Émission animée par Bernard Pivot, France 2 (2005).

http://www.encres-vagabondes.com/rencontre/yingchen.htm [consulté le 18/06/2012]

\section{Annexe : Fiche pédagogique élaborée par Béatrice Bouvier et Anne Prouteau}

Publiée dans Français 2000. 206-207 (avril 2007) : 76-83.

\section{L'univers singulier de François Cheng \\ Présentation de l'auteur}

Poète, calligraphe, traducteur des plus grands poètes français, François Cheng est né en Chine en 1929. Il a été élu à l'académie française en 2002. Arrivé en France pour faire des études à dix-neuf ans grâce à une bourse, il ne connaissait pas un mot de français. Il a fréquenté l'école des hautes études en Sciences sociales comme étudiant avant de devenir assistant de Gaston Berger puis bien plus tard professeur à l'université. N'ayant pas pu rentrer dans son pays qui entre temps s'était fermé, il vit les affres de l'exil et mène le chemin solitaire des études et de l'apprentissage. Au cours de ses années studieuses, il rencontre des sinologues et des intellectuels (R. Barthes, J. Kristeva, J. Lacan, G. Deleuze, E. Levinas, S. Leys...) qui l'encouragent et apprécient son travail. Dans les années 70, il publie L'écriture poétique chinoise et Vide et plein, le langage pictural chinois qui marquent un tournant dans l'étude sinologique. Après de longues années de contradictions et de déchirements, il opte finalement pour la langue française comme outil de création, tandis que sa langue maternelle, mise en sourdine, reste une source d'inspiration et une muse qui nourrit son imaginaire. Il vit son bilinguisme comme une aventure linguistique douloureuse et passionnante et se voit comme un « passeur », qui tente d'instaurer un dialogue entre l'Occident et la Chine.

\section{Résumé du roman}

Dao-Sheng était un jeune musicien de vingt ans lorsqu'il a aperçu Lan-Ying, «Fine Orchidée », une jeune fille de condition sociale plus élevée que lui. Cet échange de regard fera naitre leur amour. Mais elle était fiancée à un seigneur local qui s'arrange pour faire envoyer le jeune homme au bagne. Ils sont séparés pendant plus de trente ans, jusqu'à ce que Dao-Sheng décide de parcourir la distance qui les sépare. Il quitte le monastère taoïste où il a appris la médecine et la divination et part la rejoindre. Lan-Ying, de son côté s'étiole, épouse 
délaissée minée par les chagrins et les épreuves. Dao-Sheng pense pouvoir se rapprocher d'elle et lui déclarer son amour en la soignant. Dans leur situation, le plus qu'ils puissent espérer c'est de se tenir la main, d'échanger furtivement un sourire mais qu'importe... ils ont l'éternité pour eux.

\section{Propos de l'auteur sur L'éternité n'est pas de trop}

«La passion amoureuse est une voie royale par laquelle l'être humain peut accéder à l'infini. »

«La nature n'est pas un décor mais la part vitale de notre esprit. La nature nous initie à la beauté et à la vie tout en incarnant ce que notre esprit porte en lui. »

«Au bout de tant d'années, ils tentent de se rejoindre comme deux constellations éloignées que la course du ciel remet en présence. »

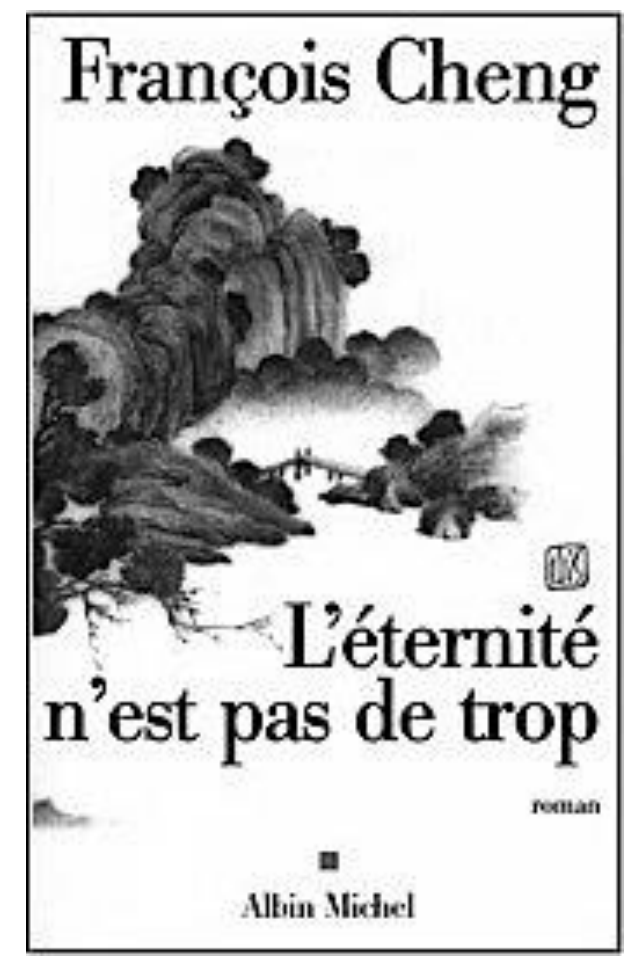

a. Que pouvez-vous dire du nom et du prénom de l'auteur ? Quelle est, d'après vous, la relation de l'auteur avec la France, avec la Chine?

b. Qu'évoque pour vous l'illustration de cette couverture de roman ?

c. Lisez le titre et choisissez la définition qui convient pour «éternité » :

1. période de temps qui n'a pas de fin $\square$ 2. période de temps qui a une fin mais pas de commencement

d. Parmi ces mots choisissez ceux qui sont de la même famille que «éternité ».
1. éternel
2. éternellement
3. étendre
4. éternuer
5. éterniser $\square$ 
e. Lisez cette phrase et cochez deux mots qui ont le même sens que le premier « éternelle » : «Plutôt l'éternelle vivacité que la vie éternelle» permanente $\square$ correspondante $\square$ continuelle $\square$ brève $\square$

g. Comment comprenez-vous cette phrase de Nietzche ?

h. Comment comprenez-vous ce titre : L'éternité n'est pas de trop ? Proposez une phrase qui a le même sens.

«A ces mots, Lan-Ying ouvre sa paume et laisse Dao-Sheng y coller la sienne. Instant de muette communion et d'extase hors paroles. L'intimité née de deux mains en symbiose est bien celle même de deux visages qui se rapprochent, ou de deux cœurs qui s'impriment l'un dans l'autre. La corole à cinq pétales, quand elle éclot, est un gant retourné de l'intérieur vers l'extérieur, elle livre son fond secret, se laisse effleurer par la brise tiède qui sans cesse passe, ou butiner sans fin par d'avides papillons et abeilles qui accourent. Entre deux mains aux doigts noués, le moindre frémissement bruit de battements d'ailes, la moindre pression provoque une onde qui s'élargit de cercle en cercle. La main, ce digne organe de la caresse, ce qu'elle caresse ici n'est pas seulement une autre main, mais la caresse même de l'autre. Caressant réciproquement la caresse, les deux partenaires basculent dans un état d'ivresse qui a peut-être été rêvé dans l'enfance, ou alors dans une avant-vie. Les veines entremêlées irriguant le désir se relient aux racines profondes de la vie; les lignes entrecroisées qui prédisent le destin tendent vers le lointain, jusqu'à rejoindre l'infini des étoiles.

L'éternité n'est pas de trop, François Cheng, 2002, Albin Michel, page 85.

\section{Activité 1}

Lisez le texte et cochez la réponse qui convient dans le tableau.

\begin{tabular}{|l|l|l|l|}
\hline & VRAI & FAUX & $\begin{array}{c}\text { ON NE SAIT } \\
\text { PAS }\end{array}$ \\
\hline $\begin{array}{l}\text { 1. Lan-Ying et Dao-Sheng se connaissaient avant cette } \\
\text { rencontre. }\end{array}$ & & & \\
\hline 2. Lan-Ying et Dao-Sheng s'embrassent. & & & \\
\hline 3. Lan-Ying et Dao-Sheng se caressent les mains. & & & \\
\hline $\begin{array}{l}\text { 4. Lan-Ying et Dao-Sheng ont une longue } \\
\text { conversation. }\end{array}$ & & & \\
\hline 5. Les deux personnages sont amoureux. & & & \\
\hline
\end{tabular}




\begin{tabular}{|l|l|l|l|}
\hline 6. La scène se passe en Chine & & & \\
\hline 7. La scène se passe à une époque ancienne & & & \\
\hline
\end{tabular}

\section{Activité 2}

Marquez les paragraphes de 1 à 4 sur votre texte et associez un titre à chaque paragraphe.

Paragraphe (1) [À ces mots... l'autre]

Paragraphe (2) [La corole... accourent]

Paragraphe (3) [Entre deux mains... l'autre]

Paragraphe (4) [Caressant... étoiles]
(1 La fleur, objet de comparaison

2 L'ouverture au monde

3 De la main au cœur

4 La caresse

Approche détaillée

\section{Activité 3}

a. Retrouvez dans le texte un synonyme de «communion ».

b. Choisissez les verbes du texte qui nécessitent une relation entre deux éléments pour agir.

1. entremêler $\square$ 2. entrecroiser $\square$ 3. éclore $\square$ 4. basculer $\square$ 5. se relier $\square$

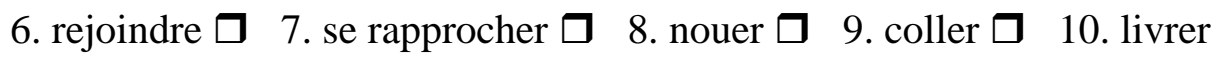

c. À votre tour, proposez ou composez des verbes qui commencent par « entre ».

\section{Activité 4}

a. Parmi les cinq sens, c'est le toucher qui est le plus présent dans ce texte. Retrouvez quatre verbes ou noms qui illustrent ce sens.

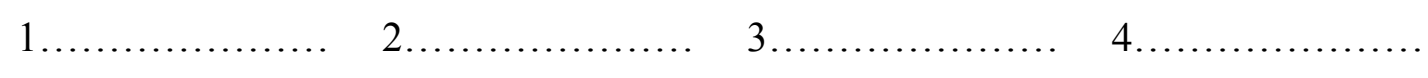

b. Que représente la corole à cinq pétales ?

c. Retrouvez l'autre métaphore de cette phrase.

d. Pourquoi, d'après vous, l'auteur a-t-il choisi de s'exprimer par métaphores ?

e. Que représentent ces métaphores ?

f. Lisez cette métaphore et à votre tour, proposez une ou deux métaphores.

«Homme libre, toujours tu chériras la mer! La mer est ton miroir. » Charles Baudelaire 


\section{Activité 5}

a. Quel est le temps utilisé par le narrateur dans cet extrait ?

b. Associez chacune des phrases à une valeur du présent.

\begin{tabular}{|l|l|l|}
\hline & $\begin{array}{c}\text { Action immédiate, } \\
\text { ponctuelle } \\
\text { (Expérience des deux } \\
\text { personnages) }\end{array}$ & $\begin{array}{c}\text { Définit une vérité } \\
\text { générale (Présent de } \\
\text { généralisation) }\end{array}$ \\
\hline $\begin{array}{l}\text { Lan-Ying ouvre sa paume. } \\
\text { un état d'ivresse. }\end{array}$ & \\
\hline $\begin{array}{l}\text { L'intimité née de deux mains en } \\
\text { symbiose est bien... }\end{array}$ & & \\
\hline Elle livre son fond secret. & & \\
\hline
\end{tabular}

\section{Activité 6}

a. Que remarque-t-on dans ce passage?

b. Soulignez et classez dans ce tableau tous les termes relatifs à la caresse.

«La main, ce digne organe de la caresse, ce qu'elle caresse ici n'est pas seulement une autre main, mais la caresse même de l'autre. Caressant réciproquement la caresse les deux partenaires basculent dans un état d'ivresse qui a peut-être été rêvé dans l'enfance, ou alors dans une avant-vie. »

\begin{tabular}{|l|l|}
\hline Verbe & \\
\hline Nom (substantif) & \\
\hline Participe présent & \\
\hline
\end{tabular}

c. Lisez le commentaire de Claude Simon, grand romancier du $\mathrm{XX}^{\mathrm{e}}$ siècle et dites en quoi l'utilisation du participe présent dans cet extrait est vraiment appropriée.

«L'emploi du participe présent me permet de me placer hors du temps conventionnel. [...] il n'y a ni fin, ni commencement dans un souvenir. »

d. Dans la phrase «La main, ce digne organe de la caresse... », que représente « ce digne organe »?

e. À votre tour proposez deux périphrases :

La main, Le cœur, 


\section{Activité 7}

a. Quelles sont les caractéristiques de cette forme d'amour ? Quelles autres grandes histoires d'amour connaissez-vous qui possèdent ces caractéristiques ?

b. Donnez un titre à cet extrait.

\section{Production écrite}

\section{Activité 8}

Imaginez ce que dit Dao-Sheng à Lan-Ying juste avant cette scène (10 à 15 lignes). Vous n'oublierez pas d'introduire une métaphore et une périphrase dans votre production.

\section{NOTES}

${ }^{1}$ Dai Sijie, Ying Chen, François Cheng, Gao Xingjian, Shan Sa, Wei Wei, Sheng Dali, Ya Ding et Ling Xi

${ }^{2}$ À l'exception de François Cheng qui publie des ouvrages sur l'art et des poèmes en chinois.

${ }^{3}$ Voir la reproduction de la première de couverture en annexe.

${ }^{4}$ L'Écriture poétique chinoise, Seuil, 1977 et 1996, Vide et plein : le langage pictural chinois, Seuil, 1979.

${ }^{5}$ Voir l'analyse détaillée d'un extrait de L'Éternité n'est pas de trop reproduite en annexe.

${ }^{6}$ Robert Van Gulick, dix-sept volumes publiés en anglais dans les années 1950, puis en français chez 10/18, dans la collection « Grands détectives » dans les années 1980 qui ont connu un important succès.

${ }^{7} \ll S a »$ signifie ou se prononce comme « sable . 